The authors have shown the 8 years clinical follow-up analyzing different treatment modalities for localized prostate cancer. I believe this assessment should be followed by health care providers managing patients with prostate cancer so we can better serve our patients.

Dr. Fernando J. Kim

Chief of Urology, Denver Health Med. Ctr. Associate Professor, Univ. Colorado Health Sci. Ctr. Director of Minimally Invasive Urol. Oncology, UCHSC

Denver, Colorado, USA

E-mail:fernando.kim@dhha.org

\title{
IMAGING
}

doi: 10.1590/S1677-55382010000700019

\section{Positive predictive value of CT urography in the evaluation of upper tract urothelial cancer}

Sadow CA, Wheeler SC, Kim J, Ohno-Machado L, Silverman SG

Department of Radiology, Brigham and Women's Hospital, Boston, MA 02115, USA

AJR Am J Roentgenol. 2010; 195: W337-43

Objective: The purpose of this study was to determine the positive predictive value of CT urography in the diagnosis of upper tract urothelial malignancies.

Materials and Methods: Retrospective review of the records of patients who underwent 2,602 CT urographic examinations revealed that $81(3 \%)$ examinations of 77 patients had findings suggesting upper tract urothelial cancer. Two radiologists in consensus categorized the findings as large masses $(>5 \mathrm{~mm})$, small masses $(\leq 5$ $\mathrm{mm}$ ), or urothelial thickening. The positive predictive value of CT urography was determined with the findings at pathologic examination $(n=42)$, followup imaging $(n=29)$, or clinical follow-up alone $(n=5)$. One patient with insufficient follow-up information was excluded. The effects of age, sex, indication for examination, imaging appearance, and urine cytology were analyzed with the Fisher's exact test or Student's $t$ test. Multivariate logistic regression analysis was used to generate a model for predicting the probability of the presence of upper tract urothelial cancer in patients with positive CT urographic examinations.

Results: The positive predictive value of CT urography for upper tract urothelial cancer was 53\% (40/76) overall, $83 \%$ (29/35) for large masses, $0 \%(0 / 17)$ for small masses, and $46 \%$ (11/24) for urothelial thickening. Imaging appearance, urine cytology, and age were significant univariate predictors $(\mathrm{p}<0.05)$ of the presence of upper tract urothelial cancer in patients with positive $\mathrm{CT}$ urographic examinations. The independent variables most likely associated with upper tract urothelial cancer were urine cytology (odds ratio, 60.0; 95\% CI, 5.5-653.7) and imaging appearance (odds ratio, 24.4; 95\% CI, 3.0-201.9) after adjusting for age and clinical indication. Conclusion: The positive predictive value of CT urography for upper tract urothelial cancer is moderate because benign findings mimic cancer. Positive findings on a CT urogram are more likely to indicate cancer in the setting of large masses or positive urine cytology.

\section{Editorial Comment}

Several studies have been shown that multidetector computerized tomography urography (CT urography) is more sensitive, specific and accurate than excretory urography in the diagnosis of upper urinary tract 
transitional cell carcinoma in patients with hematuria and in patients with history of urothelial cancer. In this retrospective study the authors shows that positive predictive value (PPV) of abnormal findings suspicious for upper tract urothelial cancer on CT urography was only moderate, that is $53 \%$ (of 76 patients with either minimally or highly suspicious findings, only 40 had pathologically proved upper tract urothelial cancer). CT urography findings suspicious for urothelial carcinoma were classified in three main categories: large mass (lesions $>5 \mathrm{~mm}$ in maximum diameter), small mass (lesions $5 \mathrm{~mm}$ in maximum diameter), or urothelial thickening. For findings classified as large masses, the PPV was $83 \%$ and for small masses was $0 \%$. We have to consider however, the large number and the variety of false-positive findings in this study. This was probably related to the retrospective analysis of reported findings. False positive findings were caused mainly by normal or hypertrophied papilla, blood clot and inflammation. Usually nonenhancing blood clots may be differentiated from enhancing urothelial tumor by comparing the findings between non-contrast phase and nephrographic phase. Presence of mild, homogeneously enhanced and thickened pelviocalyceal urothelium is relatively frequent feature of patients with symptomatic or asymptomatically urinary tract infection. Normal prominent renal papillae may occasionally invaginate deeply into the calices and thus simulate urothelial tumor. The awareness of such anatomic variation and the search for this finding in other papillae in the same patient, are helpful for the adequate diagnosis.

The authors of this manuscript, however, offered important information regarding the value of urine cytology studies, which were available in $80 \%$ of patients. Urine cytology was very important for the adequate characterization of pelviocalyceal abnormalities, such as urothelial tumor. When urine cytology was suspicious or malignant and an upper tract urothelial abnormality was found at CT urography, the PPV for upper tract urothelial carcinoma was $92 \%$.

Dr. Adilson Prando

Head, Department of Radiology and Diagnostic Imaging, Vera Cruz Hospital

Campinas, São Paulo, Brazil

E-mail: adilson.prando@gmail.com

doi: $10.1590 / S 1677-55382010000700020$

Urinary calculi composed of uric acid, cystine, and mineral salts: differentiation with dual-energy $\mathrm{CT}$ at a radiation dose comparable to that of intravenous pyelography

Thomas C, Heuschmid M, Schilling D, Ketelsen D, Tsiflikas I, Stenzl A, Claussen CD, Schlemmer HP

Department of Diagnostic and Interventional Radiology, University of Tübingen, Tübingen, Germany

Radiology. 2010; 257: 402-9

Purpose: To retrospectively evaluate radiation dose, image quality, and the ability to differentiate urinary calculi of differing compositions by using low-dose dual-energy computed tomography (CT).

Materials and Methods: The institutional review board approved this retrospective study; informed consent was waived. A low-dose dual-energy CT protocol (tube voltage and reference effective tube current-time product, $140 \mathrm{kV}$ and $23 \mathrm{mAs}$ and $80 \mathrm{kV}$ and $105 \mathrm{mAs}$; collimation, $64 \times 0.6 \mathrm{~mm}$; pitch, 0.7) for the detection of urinary calculi was implemented into routine clinical care. All patients $(n=112)$ who were examined with this protocol from July 2008 to August 2009 were included. The composition of urinary calculi was assessed by using 